\title{
Note on the Pair-crossing Number and the Odd-crossing Number
}

\author{
Géza Tóth
}

Received: 18 December 2006 / Revised: 10 April 2007 /

Published online: 14 September 2007

(C) Springer Science+Business Media, LLC 2007

\begin{abstract}
The crossing number $\mathrm{CR}(G)$ of a graph $G$ is the minimum possible number of edge-crossings in a drawing of $G$, the pair-crossing number PAIR-CR $(G)$ is the minimum possible number of crossing pairs of edges in a drawing of $G$, and the oddcrossing number ODD-CR $(G)$ is the minimum number of pairs of edges that cross an odd number of times. Clearly, ODD-CR $(G) \leq \operatorname{PAIR-CR}(G) \leq \mathrm{CR}(G)$. We construct graphs with $0.855 \cdot$ PAIR-CR $(G) \geq$ ODD-CR $(G)$. This improves the bound of Pelsmajer, Schaefer and Štefankovič. Our construction also answers an old question of Tutte.
\end{abstract}

Slightly improving the bound of Valtr, we also show that if the pair-crossing number of $G$ is $k$, then its crossing number is at most $O\left(k^{2} / \log ^{2} k\right)$.

Keywords Drawings of a graph · Crossing number

\section{Introduction}

In a drawing of a graph $G$ vertices are represented by points and edges are represented by Jordan curves connecting the corresponding points. If it does not lead to confusion, we do not make any notational distinction between vertices (resp. edges) and points (resp. curves) representing them. We assume that the edges do not pass through vertices, any two edges have finitely many common points and each of them is either a common endpoint, or a proper crossing. We also assume that no three edges cross at the same point.

The crossing number $\mathrm{CR}(G)$ is the minimum number of edge-crossings (i.e. crossing points) over all drawings of $G$. The pair-crossing number PAIR-CR $(G)$ is the

G. Tóth's research was supported by the Hungarian Research Fund grant OTKA-K-60427 and the Research Foundation of the City University of New York.

G. Tóth $(\bowtie)$

Rényi Institute, Hungarian Academy of Sciences, Budapest, Hungary

e-mail: geza@renyi.hu 
minimum number of crossing pairs of edges over all drawings of $G$, and the oddcrossing number ODD-CR $(G)$ is the minimum number of pairs of edges that cross an odd number of times over all drawings of $G$.

Clearly, for any graph $G$ we have

$$
\operatorname{ODD}-\mathrm{CR}(G) \leq \operatorname{PAIR}-\mathrm{CR}(G) \leq \mathrm{CR}(G) .
$$

Pach and Tóth [1] proved that $\mathrm{CR}(G)$ cannot be arbitrarily large if ODD-CR $(G)$ is bounded, namely, for any $G$, if $\operatorname{ODD}-\mathrm{CR}(G)=k$, then $\operatorname{CR}(G) \leq 2 k^{2}$ and this is the best known bound. Obviously it follows that PAIR-CR $(G) \leq 2 k^{2}$ as well and this is also the best known bound. On the other hand, Pelsmajer, Schaefer and Štefankovič [4] proved that ODD-CR $(G)$ and PAIR-CR $(G)$ are not necessarily equal, they constructed a series of graphs with

$$
\operatorname{ODD}-\mathrm{CR}(G)<\left(\frac{\sqrt{3}}{2}+o(1)\right) \cdot \operatorname{PAIR}-\mathrm{CR}(G) .
$$

We slightly improve their bound with a completely different construction.

Theorem 1 There is a series of graphs $G$ with

$$
\operatorname{ODD}-\mathrm{CR}(G)<\left(\frac{3 \sqrt{5}}{2}-\frac{5}{2}+o(1)\right) \cdot \operatorname{PAIR}-\mathrm{CR}(G)
$$

Note that $\frac{\sqrt{3}}{2} \approx 0.866$ and $\frac{3 \sqrt{5}}{2}-\frac{5}{2} \approx 0.855$. There are many other versions of the crossing number (see e.g. [2, 3]). Tutte [6] defined the following version which we call independent algebraic crossing number, $\operatorname{IALG-CR}(G)$, and we also define its close relative the algebraic crossing number, $\operatorname{ALG-CR}(G)$.

Orient the edges of $G$ arbitrarily. For any drawing $D$ of $G$, and any two edges $e$ and $f$, let $c^{+}$(resp. $c^{-}$) be the number of $e-f$ crossings where $e$ crosses $f$ from left to right (resp. from right to left). Let $c(e, f)=\left|c^{+}-c^{-}\right|$, and let $c(D)=\sum c(e, f)$ where the summation is for all pairs of independent edges. Similarly, let $c^{\prime}(D)=$ $\sum c(e, f)$ where the summation is for all pairs of edges. Finally, let IALG-CR $(G)$ be the minimum of $c(D)$ for all drawings $D$ of $G$, and let ALG-CR $(G)$ be the minimum of $c^{\prime}(D)$ for all drawings $D$ of $G$.

It is easy to see that for any graph $G$ we have $\operatorname{IALG-CR}(G) \leq \mathrm{ALG}-\mathrm{CR}(G)$ and

$$
\operatorname{ODD}-\mathrm{CR}(G) \leq \mathrm{ALG}-\mathrm{CR}(G) \leq \mathrm{CR}(G)
$$

In the construction of Pelsmajer, Schaefer and Štefankovič [4] for each of the graphs the pair-crossing number and the algebraic crossing number are equal. Therefore, for their series of graphs

$$
\operatorname{ODD}-\mathrm{CR}(G)<\left(\frac{\sqrt{3}}{2}+o(1)\right) \cdot \operatorname{ALG}-\mathrm{CR}(G) .
$$

We show that $\operatorname{ALG}-\mathrm{CR}(G)$ and $\operatorname{PAIR-CR}(G)$ are not necessarity equal either. 
Theorem 2 There is a series of graphs $G$ with

$$
\operatorname{ALG}-\mathrm{CR}(G)<\left(\frac{3 \sqrt{5}}{2}-\frac{5}{2}+o(1)\right) \cdot \operatorname{PAIR}-\mathrm{CR}(G)
$$

Since ODD-CR $(G) \leq \operatorname{ALG-CR}(G)$ for every graph $G$, Theorem 1 is an immediate consequence of Theorem 2. Tutte [6] asked if IALG-CR $(G)=\mathrm{CR}(G)$ holds for every graph $G$. Since IALG-CR $(G) \leq \operatorname{ALG-CR}(G)$, Theorem 2 gives a negative answer for this question. Finally, since $\operatorname{PAIR-CR}(G) \leq \mathrm{CR}(G)$, Theorems 1 and 2 hold also for $\mathrm{CR}(G)$ instead of PAIR-CR $(G)$. Moreover, the whole argument works, without any change.

It is still a challenging open question whether $\operatorname{CR}(G)=\operatorname{PAIR-CR}(G)$ holds for all graphs $G$. Pach and Tóth [1] proved that for any $G$, if PAIR-CR $(G)=k$, then $\mathrm{CR}(G) \leq 2 k^{2}$. Valtr [7] managed to improve this bound to $\mathrm{CR}(G) \leq 2 k^{2} / \log k$. Based on the ideas of Valtr, in this note we give a further little improvement.

Theorem 3 For any graph $G$, if $\operatorname{PAIR-CR}(G)=k$, then $\operatorname{CR}(G) \leq 9 k^{2} / \log ^{2} k$.

\section{Proof of Theorem 2}

The Idea and Sketch of the Construction For simplicity, we write alg-crossing number for the algebraic crossing number. In the description we use weights on the edges of the graph. If we substitute each weighted edge by an appropriate number of parallel paths, say, each of length two, we can obtain an unweighted simple graph whose ratio of the pair-crossing and alg-crossing numbers is arbitrarily close to that of the weighted construction.

First of all, take a "frame" $F$, which is a cycle $K$ with very heavy edges, together with a vertex $V$ connected to all vertices of the cycle, also with very heavy edges. In the optimal drawings the edges of $F$ do not participate in any crossing, and we can assume that $V$ is drawn outside the cycle $K$. Therefore, all additional edges and vertices of the graph will be inside $K$.

We have four further vertices, each connected to three different vertices of the frame-cycle $K$. These three edges have weights $1,1, w$ respectively, with some $1<w<2$. Each one of these four vertices, together with the adjacent three edges, and the frame $F$, is called a component of the construction.

If we take any two of the components, it is easy to see how to draw them optimally, both in the alg-crossing and pair-crossing sense. See Fig. 1. The point is that if we take all four components, we can still draw them such that each of the six pairs are drawn optimally, in the alg-crossing sense. See Fig. 2. On the other hand, it is easy to see that it is impossible to draw all six pairs optimally in the pair-crossing sense, some pairs will not have their best drawing. See Fig. 3. Note that we did not indicate vertex $V$ of the frame.

We get the best result with $w=\frac{\sqrt{5}+1}{2}$. Actually, we will see that among any three components there is a pair which is not drawn optimally in the pair-crossing sense. So, we could take the union of just three components, but that gives a weaker bound. 


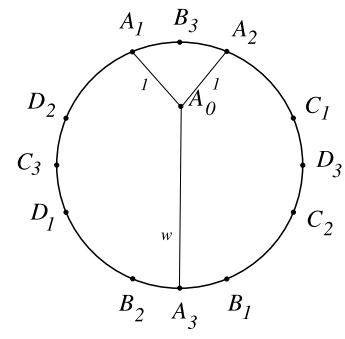

(a)

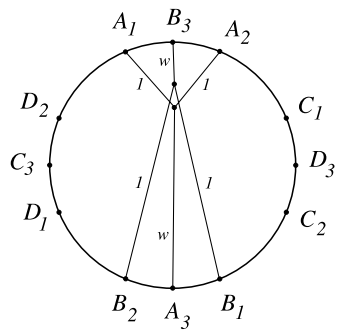

(b)

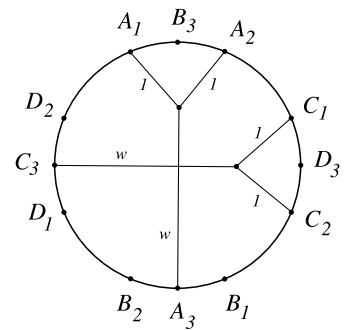

(c)

Fig. 1 a Component $A$; b, c Optimal drawings of the pairs $(A, B)$ and $(A, C)$, respectively

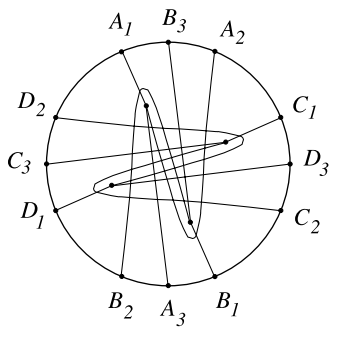

(a)

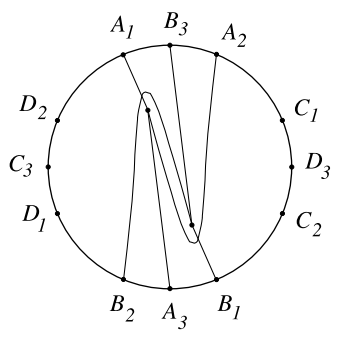

(b)

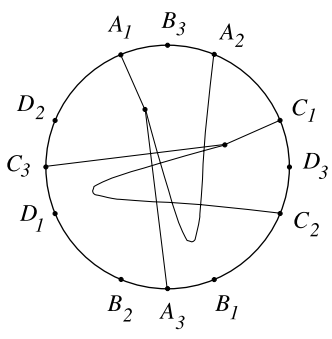

(c)

Fig. 2 a Optimal drawing of $G$ in the alg-crossing sense; b, c The pairs $(A, B)$ and $(A, C)$ respectively from the same drawing

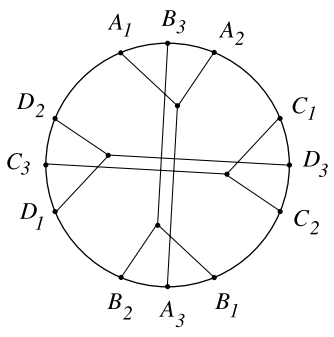

(a)

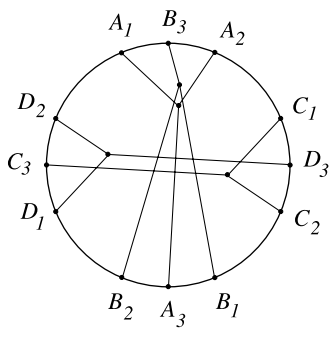

(b)

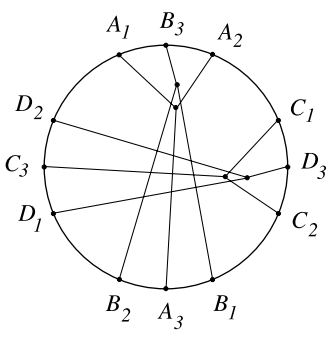

(c)

Fig. 3 a, b Cases 1 and 2 of Lemma 2, respectively, optimal drawings of $G$ in the pair-crossing sense; c Case 3, not optimal drawing

Proof of Theorem 2 A weighted graph $G$ is a graph with positive weights on its edges. For any edge $e$ let $w(e)$ denote its weight. For any fixed drawing $\mathcal{G}$ of $G$, the pair-crossing value PAIR-CR $(\mathcal{G})=\sum w(e) w(f)$ where the sum goes over all crossing pairs of edges $e, f$. For the alg-crossing value ALG-CR $(\mathcal{G})$, orient the edges of $G$ arbitrarily, let $c^{+}$(resp. $c^{-}$) be the number of $e-f$ crossings where $e$ crosses $f$ from left to right (resp. from right to left), let $c(e, f)=\left|c^{+}-c^{-}\right|$. The alg-crossing value ALG-CR $(\mathcal{G})=\sum w(e) w(f) c(e, f)$ where the sum goes over all pairs of edges $e, f$. 
The pair-crossing number (resp. alg-crossing number) is the minimum of the paircrossing value (resp. alg-crossing value) over all drawings. That is,

$$
\begin{aligned}
\operatorname{PAIR}-\mathrm{CR}(G) & =\min _{\text {over all drawings }} \sum_{\begin{array}{c}
\text { for all crossing pairs } \\
\text { of edges } e, f
\end{array}} w(e) w(f), \\
\operatorname{ALG}-\mathrm{CR}(G) & =\sum_{\text {over all drawings }} \sum_{\begin{array}{c}
\text { for all pairs } \\
\text { of edges } e, f
\end{array}} w(e) w(f) c(e, f) .
\end{aligned}
$$

Theorem 4 There exists a weighted graph $G$ with

$$
\operatorname{PAIR-CR}(G)=\left(\frac{3 \sqrt{5}}{2}-\frac{5}{2}\right) \cdot \operatorname{ALG-CR}(G) .
$$

Proof First we define the weighted graph $G$. Take nine vertices, $A_{1}, B_{3}, A_{2}, C_{1}, D_{3}$, $C_{2}, B_{1}, A_{3}, B_{2}, D_{1}, C_{3}, D_{2}$ which form cycle $K$ in this order. Vertex $V$ is connected to all of the nine vertices of $K$. These vertices and edges form the "frame" $F$. All edges of $F$ have extremely large weights, therefore, they do not participate in any crossing in an optimal drawing. We can assume without loss of generality that $V$ is drawn outside the cycle $K$, so all further edges and vertices of $G$ will be inside $K$.

There are four more vertices, $A_{0}, B_{0}, C_{0}, D_{0}$, and for $X=A, B, C, D, X_{0}$ is connected to $X_{1}, X_{2}$, and $X_{3}$. The weight $w\left(X_{0} X_{1}\right)=w\left(X_{0} X_{2}\right)=1$ and $w\left(X_{0} X_{3}\right)=$ $w=\frac{\sqrt{5}+1}{2}$. Graph $X$ is a subgraph of $G$, induced by the frame and $X_{0}$. See Fig. 1. Finally, for any $X, Y=A, B, C, D, X \neq Y$, let PAIR-CR $(X, Y)=\operatorname{PAIR-CR}(X \cup Y)$, and $\operatorname{ALG-CR}(X, Y)=\operatorname{ALG-CR}(X \cup Y)$.

First we find all these crossing numbers. Moreover, we also find the second smallest pair-crossing values.

Start with $A \cup C$. Since the path $A_{1} B_{3} A_{2}$ is not intersected by any edge in an optimal drawing, we can contract it to one vertex, without changing the pair-crossing number, so now $A_{1}=A_{2}$. Consider the edges $e_{1}=A_{1} A_{0}$ and $e_{2}=A_{2} A_{0}$. Now they connect the same vertices. Suppose that they do not go parallel in an optimal drawing. Let $w^{*}\left(e_{1}\right)$ (resp. $\left.v^{*}\left(e_{2}\right)\right)$ be the sum of the weights of the edges crossing $e_{1}$ (resp. $e_{2}$ ) and assume without loss of generality that $w^{*}\left(e_{1}\right) \leq w^{*}\left(e_{2}\right)$. Then draw $e_{2}$ parallel with $e_{1}$, the drawing obtained is at least as good as the original drawing was, so it is optimal as well. Therefore, we can assume without loss of generality that $e_{1}$ and $e_{2}$ go parallel in an optimal drawing, so we can substitute them by one edge of weight 2. Similarly, we can contract the path $C_{1} D_{3} C_{2}$ and substitute the edges $C_{1} C_{0}$ and $C_{2} C_{0}$ by one edge of weight 2 . Now we have a very simple graph, whose pair-crossing number is immediate, that is, we have two paths $C_{1} C_{0} C_{3}$ and $A_{1} A_{0} A_{3}$, which have to cross each other, and on both paths one edge has weight $w$, the other one has weight 2 . Since $w<2$, in the optimal drawing the edges $A_{0} A_{3}$ and $C_{0} C_{3}$ will cross each other and no other edges cross so we have $\operatorname{PAIR-CR}(A, C)=w^{2}$. Moreover, it is also clear that the second smallest pair-crossing value is $2 w$.

The same argument holds for $\operatorname{ALG-CR}(A, C)$, moreover, by symmetry, we can argue exactly the same way for the pairs $(A, D),(B, C)$, and $(B, D)$. 
Now we determine PAIR-CR $(A, B)$ and the second smallest pair-crossing value. The edges $a_{1}=A_{0} A_{1}, a_{2}=A_{0} A_{2}, a_{3}=A_{0} A_{3}$ divide the interior of $F$ into three regions $R_{1}, R_{2}$ and $R_{3}$. Number them in such a way that for $i=1,2,3, a_{i}$ is outside $R_{i}$. See Fig. 1. Once we place $B_{0}$ into one of these regions, it is clear how to draw the edges $b_{1}=B_{0} B B_{1}, b_{2}=B_{0} B B_{2}, b_{3}=B_{0} B B_{3}$ to get the best of the possible drawings. If $B_{0}$ is in $R_{1}$ or in $R_{2}$, we get the pair-crossing value $2 w$, but if we place $B_{0}$ in $R_{3}$, then we get 2 . Again, the same argument holds for $\operatorname{ALG-CR}(A, B)$, and by symmetry, the situation is the same with the pair $(C, D)$. See Fig. 1.

Lemma $1 \operatorname{ALG-CR}(G)=4 w^{2}+4$.

Proof We have ALG-CR $(G) \geq \operatorname{ALG-CR}(A, B)+\operatorname{ALG-CR}(A, C)+\operatorname{ALG}-\mathrm{CR}(A, D)+$ $\operatorname{ALG}-\mathrm{CR}(B, C)+\mathrm{ALG}-\mathrm{CR}(B, D)+\mathrm{ALG}-\mathrm{CR}(C, D)=4 w^{2}+4$, and there is a drawing (see Fig. 2) with exactly this alg-crossing value.

Lemma $2 \operatorname{PAIR-CR}(G)=4 w^{2}+4 w$.

Proof The argument, except for the exact calculation, should be clear from the figures. While we have a drawing which is optimal for all six pairs in the alg-crossing sense (see Fig. 2), in the pair-crossing sense some of the pairs will not be optimal, they have to take at least the second smallest pair-crossing value. We start with an observation that in any triple at least one pair is not optimal. Then we will distinguish three cases.

Take a drawing $\mathcal{G}$ of $G$. Suppose that we have a drawing $\mathcal{G}$ of $G$ where the pairs $(A, C)$ and $(A, D)$ are drawn optimally, that is, $\operatorname{PAIR-CR}(\mathcal{A}, \mathcal{C})=\operatorname{PAIR}-\mathrm{CR}(\mathcal{A}, \mathcal{D})=$ $w^{2}$. Recall that the edges $a_{1}=A_{0} A_{1}, a_{2}=A_{0} A_{2}, a_{3}=A_{0} A_{3}$ divide the interior of $F$ into three regions $R_{1}, R_{2}$ and $R_{3}$. It follows from the above argument that $C_{0} \in R_{1}$, $D_{0} \in R_{2}$. But then the pair $(C, D)$ is not drawn optimally, that is, $\operatorname{PAIR-CR}(\mathcal{C}, \mathcal{D})>2$, so we have PAIR-CR $(\mathcal{C}, \mathcal{D}) \geq 2 w$. In other words, it is impossible that all three pairs $(A, C),(A, D),(C, D)$ are drawn optimally at the same time. By symmetry, this observation holds for any triple of $A, B, C, D$.

We have to distinguish three cases.

Case 1 Neither $(A, B)$, nor $(C, D)$ are drawn optimally. In this case, $\operatorname{PAIR-CR}(\mathcal{A}, \mathcal{B})>2$ so by the above $\operatorname{argument}$ we have $\operatorname{PAIR-CR}(\mathcal{A}, \mathcal{B}) \geq 2 w$, and similarly PAIR-CR $(\mathcal{C}, \mathcal{D}) \geq 2 w$. For all other pairs we have pair-crossing value at least $w^{2}$, therefore, $\operatorname{PAIR-CR}(\mathcal{G})=\operatorname{PAIR}-\mathrm{CR}(\mathcal{A}, \mathcal{B})+\operatorname{PAIR}-\mathrm{CR}(\mathcal{A}, \mathcal{C})+$ $\operatorname{PAIR-CR}(\mathcal{A}, \mathcal{D})+\operatorname{PAIR-CR}(\mathcal{B}, \mathcal{C})+\operatorname{PAIR-CR}(\mathcal{B}, \mathcal{D})+\operatorname{PAIR}-\mathrm{CR}(\mathcal{C}, \mathcal{D}) \geq 4 w^{2}+$ $4 w$.

Case $2(A, B)$ is drawn optimally, $(C, D)$ is not. Since $(A, B)$ is drawn optimally, one of the pairs $(A, C)$ and $(B, C)$ and one of the pairs $(A, D)$ and $(B, D)$ is not drawn optimally so we have $\operatorname{PAIR-CR}(\mathcal{A}, \mathcal{C})+\operatorname{PAIR}-\mathrm{CR}(\mathcal{B}, \mathcal{C}) \geq w^{2}+$ $2 w$ and analogously $\operatorname{PAIR-CR}(\mathcal{A}, \mathcal{D})+\operatorname{PAIR}-\mathrm{CR}(\mathcal{B}, \mathcal{D}) \geq w^{2}+2 w$ therefore, $\operatorname{PAIR-CR}(\mathcal{G})=\operatorname{PAIR}-\mathrm{CR}(\mathcal{A}, \mathcal{B})+\operatorname{PAIR-CR}(\mathcal{A}, \mathcal{C})+\operatorname{PAIR}-\mathrm{CR}(\mathcal{A}, \mathcal{D})+$ $\operatorname{PAIR-CR}(\mathcal{B}, \mathcal{C})+\operatorname{PAIR}-\mathrm{CR}(\mathcal{B}, \mathcal{D})+\operatorname{PAIR}-\mathrm{CR}(\mathcal{C}, \mathcal{D}) \geq 2 w^{2}+6 w+2=4 w^{2}+4 w$ The last equality can be verified by solving the quadratic equation. 
Case 3 Both $(A, B)$ and $(C, D)$ are drawn optimally. If none of the other four pairs is optimal, then we have $\operatorname{PAIR}-\mathrm{CR}(\mathcal{G})=\operatorname{PAIR}-\mathrm{CR}(\mathcal{A}, \mathcal{B})+\operatorname{PAIR}-\mathrm{CR}(\mathcal{A}, \mathcal{C})+$ $\operatorname{PAIR-CR}(\mathcal{A}, \mathcal{D})+\operatorname{PAIR}-\mathrm{CR}(\mathcal{B}, \mathcal{C})+\operatorname{PAIR}-\mathrm{CR}(\mathcal{B}, \mathcal{D})+\operatorname{PAIR}-\mathrm{CR}(\mathcal{C}, \mathcal{D}) \geq 8 w+4=$ $4 w^{2}+4 w$. So we can assume that one of them, say $(A, C)$ is drawn optimally, that is, $\operatorname{PAIR-CR}(\mathcal{A}, \mathcal{C})=w^{2}$. Since in any triple we have at least one nonoptimal pair, we have $\operatorname{PAIR-CR}(\mathcal{B}, \mathcal{C}) \geq 2 w$ and $\operatorname{PAIR-CR}(\mathcal{A}, \mathcal{D}) \geq 2 w$. We estimate PAIR-CR $(\mathcal{B}, \mathcal{D})$ now.

Again, the edges $a_{1}=A_{0} A_{1}, a_{2}=A_{0} A_{2}, a_{3}=A_{0} A_{3}$ of $A$ divide the interior of $F$ into three regions $R_{1}, R_{2}$ and $R_{3}$ with $R_{i}$ is the one to the opposite of $a_{i}$. Similarly define the regions $Q_{1}, Q_{2}, Q_{3}$ for $C$. Since $(A, C)$ is drawn optimally, $R_{3}$ and $Q_{3}$ are disjoint. Since $(A, B)$ is drawn optimally, $B_{0} \in R_{3}$, and since $(C, D)$ is also drawn optimally, $D_{0} \in Q_{3}$. See Fig. 3. Now it is not hard to see that the edge $D_{0} D_{1}$ either crosses $A_{0} A_{1}, A_{0} A_{2}$, and $B_{0} B_{3}$, or $B_{0} B_{1}, B_{0} B_{2}$, and $A_{0} A_{3}$. The same holds for the edge $D_{0} D_{1}$, so $\operatorname{PAIR}-\mathrm{CR}(\mathcal{A}, \mathcal{D})+\operatorname{PAIR}-\mathrm{CR}(\mathcal{B}, \mathcal{D}) \geq 2 w+4$. So we have $\operatorname{PAIR-CR}(\mathcal{G})=$ $\operatorname{PAIR-CR}(\mathcal{A}, \mathcal{B})+\operatorname{PAIR-CR}(\mathcal{A}, \mathcal{C})+\operatorname{PAIR-CR}(\mathcal{A}, \mathcal{D})+\operatorname{PAIR-CR}(\mathcal{B}, \mathcal{C})+$ $\operatorname{PAIR-CR}(\mathcal{B}, \mathcal{D})+\operatorname{PAIR-CR}(\mathcal{C}, \mathcal{D}) \geq w^{2}+4 w+8>4 w^{2}+4 w$. This concludes the proof of Lemma 2.

Now we have

$$
\frac{\operatorname{ALG}-\mathrm{CR}(G)}{\operatorname{PAIR}-\mathrm{CR}(G)}=\frac{4 w^{2}+4}{4 w^{2}+4 w}=\frac{-5}{2}+\frac{3 \sqrt{5}}{2},
$$

and Theorem 4 follows immediately.

Proof of Theorem 2 Let $\varepsilon>0$ an arbitrary small number. Let $p$ and $q$ be positive integers with the property that $w\left(1+\frac{\varepsilon}{10}\right)>\frac{p}{q}>w\left(1-\frac{\varepsilon}{10}\right)$. Let $G_{\varepsilon}$ be the following graph. In the weighted graph $G$ of Theorem 4, (i) substitute each edge $e=X Y$ of weight 1 with $q$ paths between $X$ and $Y$, each of length 2, (ii) substitute each edge $e=X Y$ of weight $w$ with $p$ paths between $X$ and $Y$, each of length 2 , and (iii) substitute each edge $e=X Y$ of the frame $F$ with a huge number of paths between $X$ and $Y$, each of length 2 . Then

$$
\frac{\operatorname{ALG}-\mathrm{CR}\left(G_{\varepsilon}\right)}{\operatorname{PAIR}-\mathrm{CR}\left(G_{\varepsilon}\right)}<\frac{\operatorname{ALG}-\mathrm{CR}(G)}{\operatorname{PAIR}-\mathrm{CR}(G)}(1+\varepsilon)<\frac{-5}{2}+\frac{3 \sqrt{5}}{2}+\varepsilon .
$$

\section{Proof of Theorem 3}

Let $G$ be a graph, PAIR-CR $(G)=k$ and take a drawing of $G$ which has exactly $k$ crossing pairs of edges. Let $t$ be a parameter, to be defined later. We distinguish three types of edges. An edge $e$ is

good if it is not crossed by any other edge;

light if it is crossed by at least one and at most $t$ other edges;

heavy if it is crossed by more than $t$ other edges.

We will apply the following result of Schaefer and Štefankovič [5]. 
Fig. 4 Switch the $u v$ segment of $e$ and $f$
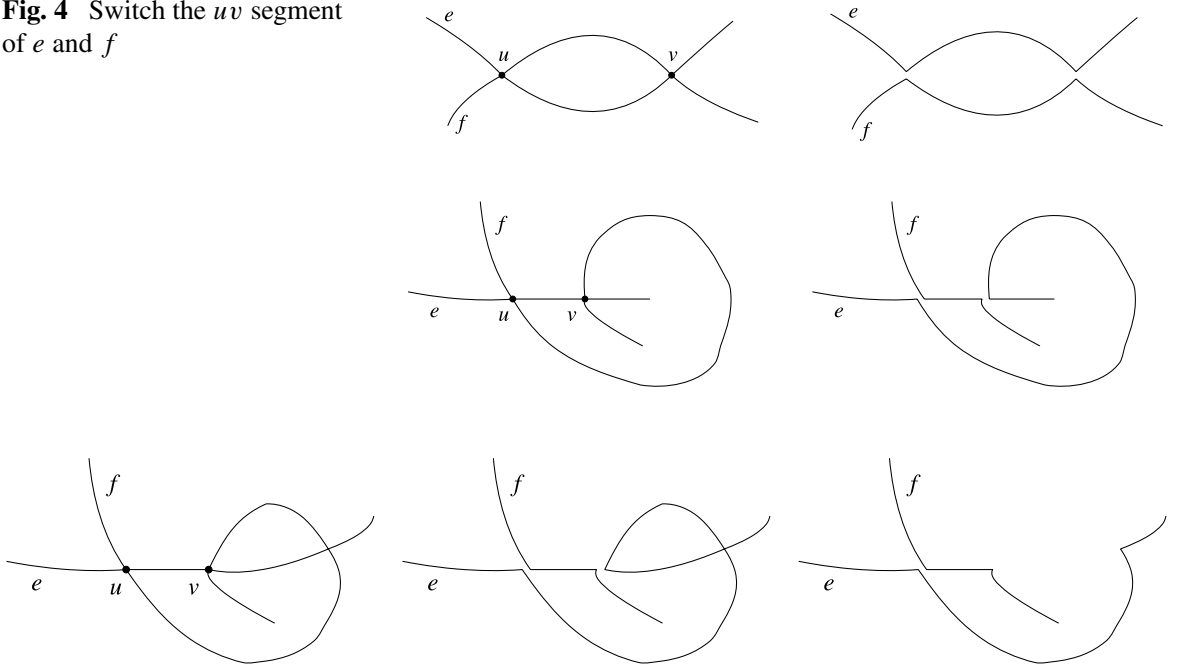

Fig. 5 Switch the $u v$ segment of $e$ and $f$ and remove the self-crossing

Lemma (Schaefer and Štefankovič [5]) Suppose that a graph is drawn in the plane, and edge $e$ is crossed by $m$ other edges. If there are at least $2^{m}$ crossings on e, then the drawing can be modified such that (i) the number of crossings between any two edges does not increase, and (ii) the number of crossings on e decreases.

Return to the proof of Theorem 3. Suppose that there is a light edge that has at least $2^{t}$ crossings. Then we can modify the drawing according to the lemma. This modification does not increase the number of crossings on any edge and does not introduce new pairs of crossing edges. On the other hand, it decreases the total number of crossings, so after finitely many applications, all light edges have less than $2^{t}$ crossings.

Now we apply two other types of redrawing steps.

Suppose that in our drawing two heavy edges $e$ and $f$ cross at least twice and let $u$ and $v$ be two crossings. Then switch the $u v$ segment of $e$ and $f$. This way (i) we reduced the number of crossings between $e$ and $f$ and (ii) the total number of crossings on any other edge remains the same.

Observe that this way we could have introduced self-crossings, in this case remove the loop formed by the self-crossing edge. This way (i) the number of crossings on any edge does not increase, and (ii) the total number of crossings decreases.

Apply the above redrawing steps as long as there are two heavy edges that cross more than once or there is a self-crossing edge. Since the total number of crossings decreases in each step, after finitely many applications any two heavy edges will cross at most once and no edge crosses itself.

Now count the number of crossings for the drawing obtained. Originally there were $k$ pairs of crossing edges. A heavy edge crosses more than $t$ other edges, so there are less than $2 k / t$ heavy edges. The total number of light edges is at most $2 k$. Each light edge has less than $2^{t}$ crossings, so the total number of crossings on the light edges is less than $2 k 2^{t}$. On the other hand, since any two heavy edges cross at 
most once, we have less than $\left(\begin{array}{c}2 k / t \\ 2\end{array}\right)$ heavy-heavy crossings. So, for the total number of crossings $C$ we have

$$
\mathrm{CR}(G) \leq C<k 2^{t+1}+\left(\begin{array}{c}
2 k / t \\
2
\end{array}\right)<k 2^{t+1}+2 k^{2} / t^{2} .
$$

Set $t=(\log k) / 2$, we obtain $\operatorname{CR}(G)<9 k^{2} / \log ^{2} k$.

Acknowledgement We are very grateful to Daniel Štefankovič for his comments.

\section{References}

1. Pach, J., Tóth, G.: Which crossing number is it anyway? J. Comb. Theory Ser. B 80, 225-246 (2000)

2. Pach, J., Tóth, G.: Thirteen problems on crossing numbers. Geombinatorics 9, 194-207 (2000)

3. Pelsmajer, M., Schaefer, M., Štefankovič, D.: Removing even crossings. In: S. Felsner (ed.) European Conference on Combinatorics, Graph Theory and Applications (EuroComb '05), DMTCS Conference Volume AE, pp. 105-110 (2005)

4. Pelsmajer, M., Schaefer, M., Štefankovič, D.: Odd crossing number is not crossing number. In: Healy, P., Nikolov, N.S. (eds.) Graph Drawing 2005. Lecture Notes in Computer Science, vol. 3843, pp. 386-396. Springer, Berlin (2006)

5. Schaefer, M., Štefankovič, D.: Decidability of string graphs. J. Comput. Syst. Sci. 68, 319-334 (2004)

6. Tutte, W.T.: Toward a theory of crossing numbers. J. Comb. Theory 8, 45-53 (1970)

7. Valtr, P.: On the pair-crossing number. In: Combinatorial and Computational Geometry. Math. Sci. Res. Inst. Publ., vol. 52, pp. 569-575. Cambridge Univ. Press, Cambridge (2005) 